\title{
Update Strategy and Model with Uncertainty of Moving Objects
}

\author{
Ya Ban ${ }^{+}$, Haiyan Sun, Xina Wang \\ School of Geodesy and Geomatics, Wuhan University, Wuhan, China
}

\begin{abstract}
Tracking moving objects is one of the most common requirements for many location-based applications. The location of a moving object changes continuously but the database location of the moving object cannot update continuously. Modelling of such moving object database should be considered to facilitate study of the performance and design parameters for this database feature. Such study is essential for selecting the optimal solution in order to minimize the implementation of the overhead cost. Location updating strategy for such type of database is the most important criteria. Spatio-temporal uncertainty is a special feature of moving objects due to the inability of precisely capturing or predicting their continuously changing locations. In this paper, we proposed a new dynamic threshold location updating strategy. The location updating threshold was given dynamically in accordance with the velocity, azimuth positioning information from the GPS. In addition, a cone model with uncertainty of current unit trajectory was presented based on thresholds. Furthermore, the model was tested through the different moving objects. The experimental results illustrated that the location update frequency can be effectively reduced, and thus the position information transmission flow and the data storage were reduced without affecting the moving objects trajectory precision.
\end{abstract}

Keywords: moving objects database, update strategies, uncertainty trajectory, uncertainty threshold.

\section{Introduction}

With the fast advance of mobile and ubiquitous computing, spatio-temporal databases[1]-[3] aiming at efficiently managing a large number of moving objects have become more realistic and attractive. Many applications, such as mobile communication systems, flight control systems, geographical information systems, transportation, traffic control, mobile resource management, and mobile workforce can benefit from the advent of spatio-temporal databases. This brought to database researchers a new challenge in the area of Moving Object Database (MOD).

Traditional Database Management System (DBMS) is not equipped to handle continuously changing data such as the transient position of moving object. This means that traditional DBMS deals with static data attributes at a given time [4], leading to a rather discrete database model. Therefore, in many MOD applications a continuous model for these dynamic objects will be essential in order to manage such moving objects [4], [5]. In this case, an updating strategy for a moving object is required. The objective of this strategy is to accurate track the current location of moving object while minimizing the number of updates. It is obvious that the more often data is updated, the more accurate the data will be. However, the cost of updating data increases with the frequency updating the data. That is, there is a trade-off between updating cost and information accuracy in designing MOD systems.

Generally speaking, the trajectories of moving objects are continuous. However, the location information is discrete and discontinuous recorded by moving object Database. When querying and visualizing moving object position and trajectory at any moment, the moving object position needs to be estimated according to the location information stored in the database. In order to simplify the study, the moving object trajectory will be defined as a straight line or curve in the time period by the proposed dynamic location update strategy.

+ Corresponding author. Tel.: + 8613554535239 .

E-mail address: banya@whu.edu.cn. 
In historical trajectory unit, cubic Hermite interpolation method is used to model trajectories and the simulation of trajectory is accurate. In current and future trajectory unit, linear function is often used to simulate trajectories, and the simulation is rough, which often leads to more error.

To address these challenges, we propose solutions to process these problems. In this paper, we propose an updating strategy of the moving objects trajectories, which is adapted to the change of velocity and direction. Non-linear index regression equation of moving object based on one parameter is established by a sliding window of velocity and direction interval. Thresholds can be adjusted dynamically taking advantage of regression parameter. In order to capture uncertainty of current trajectory, we model the trajectory as a cone shaped volume in 3D. Traditionally, spatio-temporal range queries ask for the objects that are inside a particular region, during a particular time interval. However, based on this model one may query the objects that will be inside the region sometime during the time interval, or for the ones that will be always inside during the time interval. Similarly, one may query the possibility that the objects will be inside the region.

Our main contributions can be summarized as follows:

(1)We propose an updating strategy of moving objects trajectories, adapted to the change of velocity and direction.

(2)We introduce a current trajectory unit model with uncertainty and a set of operators for query with uncertainty.

The paper is organized as follows: Section 2 discusses the related work on strategies for updating and spatio-temporal uncertainty models. Section 3 introduces our updating policies for trajectories. Section 4 introduces our model of moving objects with uncertainty and operators for query with uncertainty. Section 5 presents experimental results. Section 6 draws some conclusions and discusses future work.

\section{Related Work}

Previous research on reducing the cost of updating the location of moving objects can be classified into three categories. Research in the first category[6]-[8] tracks the object locations only. Objects move in the Euclidean space and the curves represent the trajectories of moving objects. One update strategy is that each moving object reports its location to the database server every timestamps, and the other strategy is to update the location every moving distance units. These two strategies are inefficient because the velocity, including speed and direction, of moving objects is not taken into consideration. For instance, an object moving with high speed may incur a large number of location updates as the time required to move $d$ distance units is short. In order to alleviate the problem of frequent updates, motion models are adopted in research of the second category $[9,10]$ to predict the location of a moving object. As each object is aware of its real location obtained from a Global Positioning System (GPS) device, a location update is issued to the database server only when the deviation between the real and predicted locations exceeds a given threshold $\mathrm{T}$. As long as the object's real location is within a circle centered at the predicted location with radius $\mathrm{T}$, its location information stored in the server does not need to be updated. This category takes the distance between the real and predicted locations into consideration without direction. To further reduce the update frequency, research in the third category[11], [12]takes advantage of the object's location, speed, and direction to construct a variable-size possible region, named the 'spatio-temporal possible region', whose location and size change as time progresses. This update policy can significantly reduce the cost of communication between each object and the database server. However, it raises other crucial issues affecting the system performance even more. These issues include (1) when to update the spatio-temporal possible region of each object and (2) how to update the spatio-temporal possible region when object update occurs.

The 3D polyline model, however, only yields the trajectory that the moving object may take with the highest possibility but is often not the exact route the moving object takes in reality. Two important approaches that capture the uncertainty of movements are the 3D cylinder model in [13], [14] and the spacetime prism model in [15], [16].The cylinder model, however, assumes that the degree of uncertainty does not change between two sample points, which is not the exact case in reality. The space-time prism model represents the uncertain movement of a moving object as a volume formed by the intersection of two half cones in the 3D space. Given the maximum speed of a moving object and two positions at the beginning and 
at the end of the movement, all possible trajectories between these two points are bounded within the volume of two half cones. Space-time prisms are more efficient than the cylinder models since they reduce the uncertain volume by two thirds because of the geometric properties of cones. There are many application examples that benefit from the space-time prism model in [17], [18]. In some GPS applications, the uncertainty is represented as an error ellipse which is the projection of a space-time prism in $2 \mathrm{D}$ space. A most recent approach discusses the problem of efficient processing of spatio-temporal range queries for moving objects with uncertainty [19]. However, there are some remaining problems that are not solved. First, two models use maximum speed to calculate uncertainty threshold. Actually, motion state not only includes the speed but also the direction. Second, Error models are constructed based on the origin and the destination of the moving point, which can be regarded as an error model for historical trajectory. No error models are established for future trajectory. We will provide solutions to the aforementioned problems in our model.

\section{Update Policies of the Moving Objects Trajectory}

Contributions to the congress are welcome from throughout the world. Manuscripts may be submitted to In the MOST model, the position of the moving object is abstracted as a function of time:

$$
\operatorname{LOC}(t)=\operatorname{LOC}\left(t_{r e f}\right)+\vec{V}\left(t-t_{r e f}\right)
$$

where $\operatorname{LOC}\left(t_{r e f}\right)$ is the location of the moving object at reference time $t_{r e f} . \vec{V}$ is current velocity of the moving object, which consists of $|V|$ and $\theta .|V|$ is the value of velocity, and $\theta$ is the direction of movement. According to Equation 1, location at any time can be calculated and location in the MOD is updated when moving object state changes.

According to the deficiencies of the update strategies above, we propose an adaptive threshold method, which is adapted to interval of velocity and direction. We take velocity dynamic threshold as an example to construct a nonlinear exponential nonlinear regression model.

$v_{1}, v_{2}, \cdots, v_{i}$ are velocity thresholds at time $t_{1}, t_{2}, \cdots, t_{i}$ and $I_{j}=v_{i+1}-v_{i}$ is the interval of velocity threshold. If $I_{j}$ increases, indicating that the state of movement is unstable, and the threshold should be reduced to increase update frequency. If $I_{j}$ decreases, indicating that the state of movement is stable and the threshold should be increased to reduce the update frequency.

The number of velocity interval $\omega$ is obtained in form of sliding window and nonlinear regression model of the moving object is constructed.

$$
\hat{I}=\hat{\alpha}_{0} e^{\hat{\alpha}_{1} j}
$$

The Equation 2 is transformed into a logarithmic form and linearization:

$$
\operatorname{In} \hat{I}=\operatorname{In} \hat{\alpha}_{0}+\hat{\alpha}_{1} j
$$

$\alpha_{0}, \alpha_{1}$ are regression coefficients. Assuming $y^{\prime}=\operatorname{In} \hat{I}, \alpha^{\prime}=\operatorname{In} \hat{\alpha}_{0}, b=\hat{\alpha}_{1}$, Equation 3 can be written as follows:

$$
y^{\prime}=\alpha^{\prime}+b j
$$

$\alpha_{0}, \alpha_{1}$ can be obtained by solution Equation 4 .

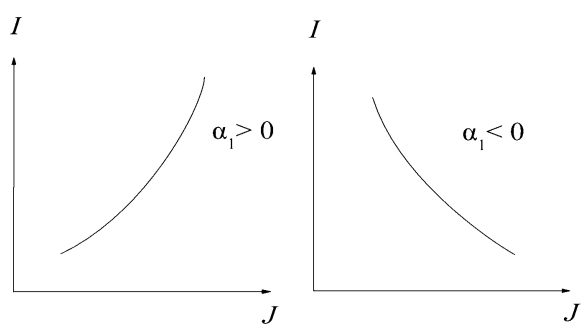

Fig. 1: Trend of interval of motion state update. 
The trend of interval of motion state update is shown in Fig. 1. If $\alpha_{1}>0$, indicating that velocity interval increases within the width of window $\omega$.If $\alpha_{1}<0$ indicating that the velocity interval decreases within the width of window $\omega$.The value of $\alpha_{1}$ is the exponential trend. The velocity threshold can be obtained from Equation 5.

$$
v_{k+1}= \begin{cases}v_{k}\left(1-\frac{1-e^{-\alpha_{1}}}{\omega}\right) & \alpha_{1}>0 \\ v_{k}\left(1+\frac{1-e^{\alpha_{1}}}{\omega}\right) & \alpha_{1} \leq 0\end{cases}
$$

The above method is used to construct a nonlinear regression model based on direction threshold, and direction update threshold is obtained as Equation 6.

$$
\theta_{j+1}= \begin{cases}\theta_{j}\left(1-\frac{1-e^{-\beta_{1}}}{\phi}\right) & \beta_{1}>0 \\ \theta_{j}\left(1+\frac{1-e^{\beta_{1}}}{\phi}\right) & \beta_{1} \leq 0\end{cases}
$$

Based on velocity and direction threshold, when moving object state change exceeds the thresholds, the trajectory of moving object is updated to MOD.

\section{Modelling the Uncertainty of Moving Objects}

In this section, we introduce a model which represents moving mbjects trajectory with uncertainty.The uncertain trajectory is obtained by associating uncertainty thresholds with each unit of the tracjectory.The moving object location will be updated to the server if it deviates from its expected moving state by velocity or direction.

Definition 1. A trajectory of a moving object is a polyline in three-dimensional space, represented as a sequence of points $\left(x_{1}, y_{1}, t_{1}\right),\left(x_{2}, y_{2}, t_{2}\right), \cdots,\left(x_{n}, y_{n}, t_{n}\right)\left(t_{1}<t_{2}<\cdots<t_{n}\right)$. For a given trajectory $\operatorname{Tr}(M)$, its projection on the XY-plane is called the trajectory of $\operatorname{Tr}(M)$.

A trajectory defines the location of a moving object as an implicit function of time. The object is at $\left(x_{i,} y_{i}\right)$ at time $t_{i}$, and during each segment $\left[t_{i}, t_{i+1}\right]$, the object moves along a straight line from $\left(x_{i}, y_{i}\right)$ to $\left(x_{i+1}, y_{i+1}\right)$, at a constant speed and direction.

Definition 2. Assuming that $\operatorname{Tr}(M)$ is the latest addition unit to trajectory ,and the the start time of the unit is tk, then the unit from tk to near future called the current unit,and moving object always moves in the current unit.

Definition 3. In the trajectories of the moving Object $M$, all the units before the current unit are called historical units.

In historical trajectory unit, cubic Hermite interpolation method is used to model the trajectory, and the simulation of trajectory is accurate. In current trajectory unit, linear function is often used to simulate trajectory, and simulation of the trajectory is rough, which often leads to more error.

Definition 4. The circle any point $(x, y, t)$ in the current unit as the center and uncertainty threshold $\mathrm{R}$ as the radius is the error region which is parallel with the $X Y$-plane.

Chapter 2 shows that, uncertainty threshold of any point in the current trajectory unit can be obtained from original point, velocity, direction and update threshold.

As shown in Fig. 2, $P_{0}\left(x_{0}, y_{0}, t_{0}\right)$ is original point. $\theta$ is direction threshold. $\alpha_{0}$ is movement azimuth of the original point. $P(x, y, t)$ is real point at time t. $P\left(x_{1}, y_{1}, t\right)$ is the predicted point at time $t . \Delta X, \Delta Y$ are coordinate error in $X$ and $Y$ direction respectively, geometric relationship shows as: 


$$
\begin{aligned}
& \Delta X=t \cdot\left[\left(v+v_{0}\right) \cdot \sin \left(\alpha_{0}+\theta\right)-v_{0} \cdot \sin \alpha_{0}\right] \\
& \Delta Y=t \cdot\left[\left(v+v_{0}\right) \cdot \cos \alpha_{0}-v_{0} \cdot \cos \left(\alpha_{0}+\theta\right)\right]
\end{aligned}
$$

Two equations in Equation 7 are squared and added, uncertainty threshold $R$ at time $\mathrm{t}$ as follows:

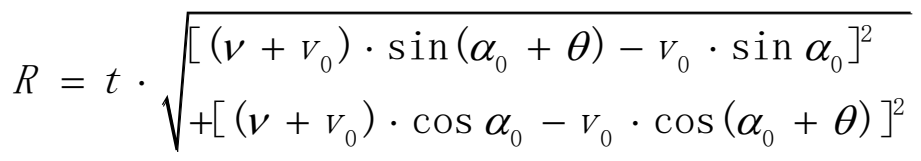

$R$ is a linear function of time show as Equation 8 .

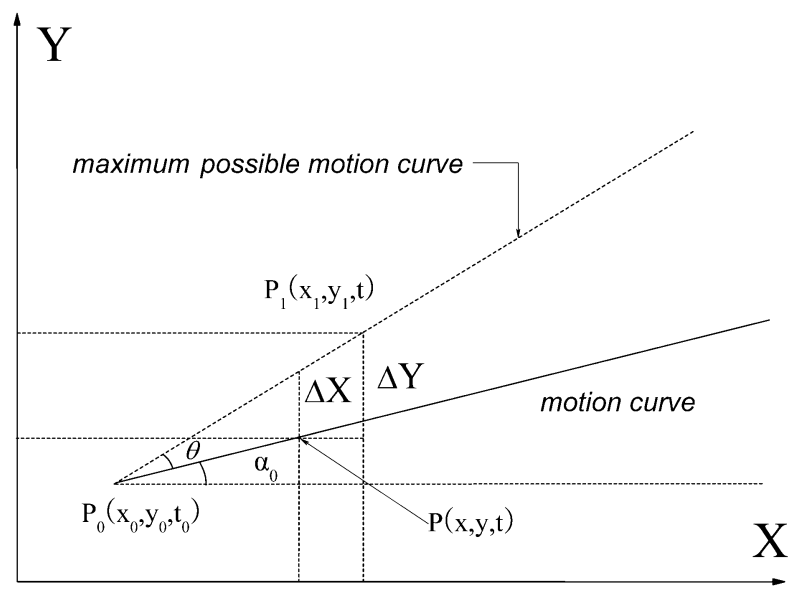

Fig. 2: Error processing.

Definition 5. A 3D cone shaped volume is formed by merged error regions from definition 4 called trajectory unit with uncertainty ,as shown in Fig. 3.

Definition 6. The projection of the 3D cone shaped volume in $X Y$-plane, is called uncertainty region of the trajectory.

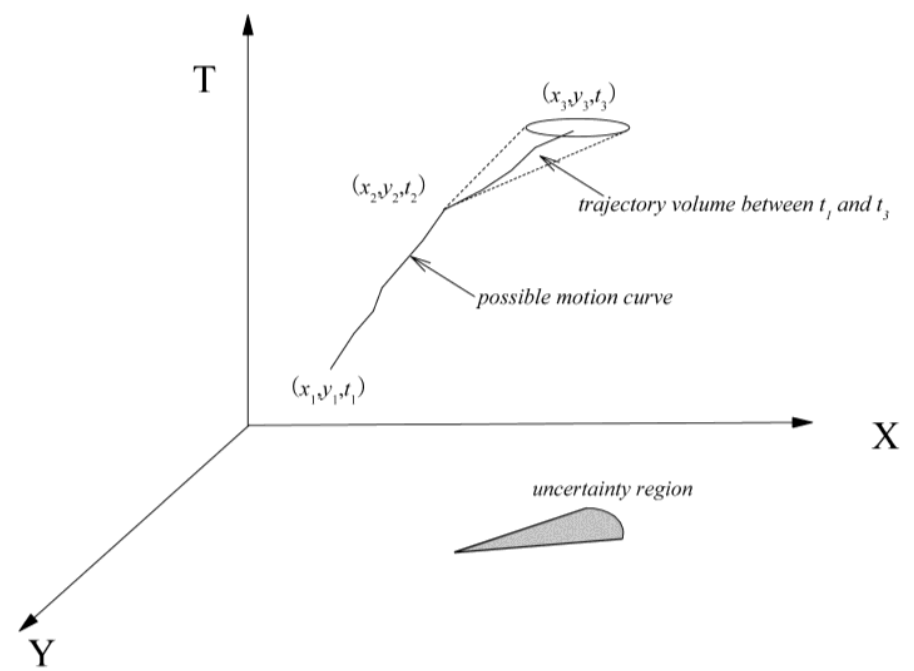

Fig. 3: Cone shaped volume representing the uncertainty of a moving object.

Shown as in Fig. 3, different point in the current unit, has different error threshold $R$, and $R$ is a positive real number, denoting the uncertainty threshold. For each point $(x, y, t)$ in the trajectory, its R-uncertainty region is a horizontal circle with radius $R$, centered at $(x, y, t)$, where $(x, y)$ is the expected location at the time $t$ $\left(t \geq t_{0}\right)$. Considering all the $t$ in 3D space, the continuous $R$-uncertainty region constitutes a 3D cone shaped volume. 


\section{Experiments}

Random motion of moving objects is tested in computer based on update strategy which moves in twodimensional space. Update frequency and deviation error of our method are compared with fixed time threshold method.

\subsection{Experimental Setup}

The experiments are conducted on a $2.93 \mathrm{GHz}$ Intel processor, which has a $8 \mathrm{~GB}$ RAM and $80 \mathrm{G}$ hard disk in XP operating system. There are several rules that the speed of moving objects is a random variable following Gaussian distribution and the direction of moving objects is a random variable following Uniformly distribution.

\subsection{Experiment Performance}

The simulated number of moving objects in the experiment is $100,200,500,1000$, and comparison cycle is 1 second, results as shown in Table 1.

Table 1: Comparison of Result Location Update

\begin{tabular}{lcccc}
\hline Number of moving objects & \multicolumn{2}{c}{ Update frequency(time/s) } & \multicolumn{2}{c}{ Mean position deviation $(\mathrm{m})$} \\
& Fixed threshold & Proposed method & Fixed threshold & Proposed method \\
\hline 100 & 0.754 & 0.611 & 7.12 & 4.52 \\
200 & 0.689 & 0.485 & 5.67 & 4.03 \\
500 & 0.701 & 0.643 & 6.35 & 3.78 \\
1000 & 0.679 & 0.523 & 6.89 & 3.81 \\
\hline
\end{tabular}

Adaptive threshold method adjusts update threshold according to state motion of the moving object update interval. Experiments demonstrate that average update frequency reduces with more accuracy compared with fixed threshold method.

\section{Conclusions and Future Work}

In this paper, we discuss the problems of trajectory update strategy and trajectory model with uncertainty. We propose an update strategy of moving objects trajectory, which is adapted to the change of velocity and direction. Non-linear index regression equation of moving object based on one parameter is established by a sliding window of velocity and direction interval. Thresholds can be adjusted dynamically taking advantage of regression parameter Experiment results show that this method not only reduces update frequency, but also improves positioning accuracy. A cone error model for current trajectory is constructed based on update thresholds and spatio-temporal query with uncertainty can be performed. In our future work, we will construct a moving object index to improve efficiency of index update and spatio-temporal query.

\section{Acknowledgments}

This work is financially supported by the National Natural Science Foundation of China (NO.91224008). One of the authors would like to thank the members of his committee for their support and the reviewers for giving the authors constructive suggestions which would help them in English and in depth to improve the quality of the paper.

\section{References}

[1] L. Forlizzi, R.H. Güting, E. Nardelli, et al. A data model and data structures for moving objects databases. International Conference on ACM Management of Data.2000,pp.319-330.

[2] R.H. Güting, M.H. Bohlen, M. Erwig, et al. A foundation for representing and querying moving objects.ACM Transactions on Database Database System.2000,25(1):1-42.

[3] Y. K. Huang, C. Lee. Efficient evaluation of continuous spatio-temporal queries on moving objects with uncertain velocity. GeoInformatica. 2010,14(2):163-200.

[4] O. Wolfson, S. Chamberlain, P. Sistla, B. Xu, J. Zhou. Domino: Databases for Moving Objects Tracking. Proceedings of the ACMSIGMOD International Conference on Management of Data. 1999, pp.547-549. 
[5] T. Murata. Petri Nets: Properties, Analysis and Application. Proceedings of the IEEE. 1989,pp.541-580.

[6] Z. Song, N. Roussopoulos. K-nearest neighbor search for moving query point. International Conference on Spatial and Temporal Databases. 2001,pp.79-96.

[7] X. Xiong, M.F. Mokbel, W.G. Aref. LUGrid: updatetolerant grid-based indexing for moving object. International Conference on Mobile Data Management. 2006, pp.13-20.

[8] X. Yu, K.Q. Pu, N. Koudas. Monitoring K-nearest neighbor queries over moving objects. International Conference on Data Engineering.2005, pp.631-642.

[9] O. Wolfson, H. Yin. Accuracy and resource consumption in tracking and location prediction. LNCS. 2003, 2750:325-343.

[10] Y. Tao, C. Faloutsos, D. Papadias, et al. Prediction and indexing of moving objects with unknown motion patterns. International Conference on ACM Management of Data.2004, pp.611-622.

[11] R. Cheng, D.V. Kalashnikov, S. Prabhakar. Querying imprecise data in moving object environments. IEEE Transactions on Knowledge and Data Engineering. 2004,16(9):1112-1127.

[12] Y. K. Huang, S.J. Liao, C. Lee. Evaluating continuous K-nearest neighbor query on moving objects with uncertainty . Information Systems. 2009, 34(4-5):415-437.

[13] G. Trajcevski, O. Wolfson, F. Zhang, S. Chamberlain.The geometry of uncertainty in moving objects databases. In: 8th International Conference on Extending Database Technology (EDBT). 2002, pp.233-250 .

[14] G. Trajcevski, O. Wolfson, K. Hinrichs, S. Chamberlain.Managing Uncertainty in Moving Objects Databases. ACM Transactions on Database Systems (TODS) .2004,29,463-507.

[15] T. Hagerstrand. What about people in regional science. Papers in Regional Science . 1970,24, 6-21.

[16] H. Liu, M. Schneider. Querying Moving Objects with Uncertainty in Spatio-Temporal Databases. DASFAA 2011, Part I . 2011,pp.357-371.

[17] D. Pfoser, C.S. Jensen. Capturing the uncertainty of moving-object representations. In: R.H. Guting, D. Papadias, F.H. Lochovsky. SSD 1999. LNCS. 1999, 1651, pp.111-131.

[18] B. Kuijpers, W. Othman. Trajectory databases: Data models, uncertainty and complete query languages. Journal of Computer and System Sciences 2010,76(7): 538-560.

[19] G. Trajcevski, A. Choudhary, O. Wolfson, L. Ye, G. Li. Uncertain range queries for necklaces. In: Proceedings of the 2010 Eleventh International Conference on Mobile Data Management. 2010, pp. 199-208. 